\title{
Orthology and paralogy constraints: satisfiability and consistency
}

\author{
Manuel Lafond", Nadia El-Mabrouk \\ From Twelfth Annual Research in Computational Molecular Biology (RECOMB) Satellite Workshop on Com- \\ parative Genomics \\ Cold Spring Harbor, NY, USA. 19-22 October 2014
}

\begin{abstract}
Background: A variety of methods based on sequence similarity, reconciliation, synteny or functional characteristics, can be used to infer orthology and paralogy relations between genes of a given gene family $\mathcal{G}$. But is a given set $\mathcal{C}$ of orthology/paralogy constraints possible, i.e., can they simultaneously co-exist in an evolutionary history for $\mathcal{G}$ ? While previous studies have focused on full sets of constraints, here we consider the general case where $\mathcal{C}$ does not necessarily involve a constraint for each pair of genes. The problem is subdivided in two parts: (1) Is $\mathcal{C}$ satisfiable, i.e. can we find an event-labeled gene tree $\mathrm{G}$ inducing $\mathcal{C}$ ? (2) Is there such a $\mathrm{G}$ which is consistent, i.e., such that all displayed triplet phylogenies are included in a species tree?
\end{abstract}

Results: Previous results on the Graph sandwich problem can be used to answer to (1), and we provide polynomial-time algorithms for satisfiability and consistency with a given species tree. We also describe a new polynomial-time algorithm for the case of consistency with an unknown species tree and full knowledge of pairwise orthology/paralogy relationships, as well as a branch-and-bound algorithm in the case when unknown relations are present. We show that our algorithms can be used in combination with ProteinOrtho, a sequence similarity-based orthology detection tool, to extract a set of robust orthology/paralogy relationships.

\section{Introduction}

Gene families, usually constructed from sequence similarity, group homologous genes, i.e., genes sharing a common ancestry: starting from a single gene copy, a history of speciations, duplications and losses is assumed to be at the origin of the observed set of extant genes. Deciphering the orthology (divergence following a speciation) and paralogy (divergence following a duplication) relations between pairs of genes inside a gene family is important and lies at the heart of many genomics studies. The reconstruction of species trees for example is usually based on the selection and alignment of orthologous gene copies. From a functional point of view, orthologs are believed to be more likely similar in function than paralogs [1]. Orthology/paralogy information is often derived from a reconciliation approach (first introduced

\footnotetext{
* Correspondence: lafonman@iro.umontreal.ca

Department of Computer Science and Operational Research, University of Montreal, Chemin de la Tour, H3C3J7 Montreal, Canada
}

by Goodman in 1979 [2]). A gene tree that best reflects the evolution of the sequences is first constructed for the gene family. Assuming a known phylogeny for the set of taxa, the non-agreement between the two trees is then explained by a set of duplication and loss events (other events such as horizontal gene transfer might also be inferred by reconciliation, although we will ignore them here). Reconciliation leads to a labeling of internal nodes of the gene tree as duplication/speciation nodes, yielding a full orthology/paralogy interpretation for each pair of genes (cf. e.g. TreeFam [3,4] used for constructing the Ensembl Compara gene trees [5], PHOG [6], MetaPHOrs [7]). This approach assumes that an accurate gene tree can be constructed for the gene family. Although inferring phylogenies is a field with a very long history, due to various limitations constructing good gene trees is still challenging. A variety of other methods have been developed for the purpose of orthology/paralogy detection. A well-known class of algorithms is based on clustering 
genes according to their sequence similarity (cf. e.g. the COG database [8], OrthoMCL [9], InParanoid [10], Proteinortho [11], orthoDB [12], eggNOG [13]). Recently, we investigated another way of detecting orthology/paralogy based on conserved synteny (conservation in gene order) $[14,15]$. Other initiatives, such as the Gene Ontology project [16], provide functional annotation that can be used as another source of orthology relations. In contrast to the reconciliation approach, only partial relations can be expected from such tree-free methods.

The orthology/paralogy information suggested by gene tree reconciliation may be contradictory with that suggested by an external source. As gene trees are known to be error-prone, more confidence can be given to such homology information when it is well-supported by various genomic observations. This raises the problem of gene tree editing based on a known set $\mathcal{C}$ of pairwise orthology/paralogy constraints. But prior to any algorithmic consideration, one should be able to state whether the set $\mathcal{C}$ is possible, i.e. whether all constraints can simultaneously co-exist in an evolutionary history of the gene family. In a recent work [14], we showed that a set of orthlogy constraints is always possible and we gave a polynomial-time algorithm for correcting a gene tree in a minimal way according to the Robinson-Foulds distance.

Recent studies have considered the connection of trees and orthology from the angle of reconstructing phylogenies from orthology relations [17-19]: How much information about the gene tree, the species tree and their reconciliation is already contained in the orthology relation between genes? In other words, having a set $\mathcal{C}$ of full pairwise orthology/paralogy relations (each pair of genes is constrained), can one reconstruct the gene and species trees? Similarly to gene tree editing, the first question to be asked is whether the orthology/paralogy constraints can simultaneously co-exist in a history of the gene family. Interestingly, by making the link with symbolic ultrametrics and co-graphs, a simple characterization of satisfiability (symbolic ultrametric) for full paralogy/ orthology relations is given in [17], where satisfiability relates to the existence of an event-labeled gene tree $G$ (symbolic representation) leading to $\mathcal{C}$. Notice that satisfiability does not mean the possibility for orthology/paralogy relations to co-exist in a true history, as the triplet phylogenies contained in $\mathrm{G}$ are not necessarily consistent (included in a species tree). The derivation of a species tree from an event-labeled gene tree is considered in [18]. Finally, the outline of a computational framework for the construction of a least resolved species tree $S$ from a set of orthology/paralogy relations, involving the extraction of maximum satisfiable relations and maximum consistent triple set is given in [19].

Here, we consider the general case for $\mathcal{C}$ : in contrast with $[17,18]$, we do not require $\mathcal{C}$ to be full, i.e., to involve a constraint for each pair of genes. We introduce the notations and problems in the following section. We then show how previous results on the Graph sandwich problem can be used to solve the problem of satisfiability. The developed algorithm for satisfiability is then adapted to the problem of consistency with a given species tree. In the case of an unknown species tree, we then study the problem of finding a gene tree that is consistent with some species tree. Finally we show in the result section how our methodology can be used, in combination with PROTEINORTHO[11], a sequence similarity-based orthology detection tool, to extract a set of robust orthology/paralogy relationships.

\section{Notations and problem statement}

In the rest of this paper, we consider a set $\Sigma$ of species and a gene family $\mathcal{G}$ where each gene $x$ belongs to a species $s(x)$ of $\Sigma$. We generalize the notation $s$ to subsets of genes: if $U \subset \mathcal{G}, s(U)=\{s(x): x \in U\}$.

As we only consider rooted trees, we will sometimes omit the word "rooted". Let $T$ be a tree. We denote by $r$ $(T)$ its root and by $L(T)$ its set of leaves. For any internal node $x$ of $T$, we denote by $T_{x}$ the subtree of $T$ rooted at $x$. We say that a node $y$ is an ancestor of $x$ if the two nodes belong to the same path from a leaf to the root of $T$, and $y$ is closer to the root. Two nodes $x$ and $y$ are unrelated if $x \neq y$ and none is the ancestor of the other. For a set of leaves $U \subseteq L(T)$, we denote by $l c a_{T}(U)$ the least common ancestral node of $U$ in $T$, i.e. the common ancestral node of the elements of $U$ which is the farthest from the root.

Let $L^{\prime}$ be a subset of $L(T)$. The restriction $\left.T\right|_{L^{\prime}}$ of $T$ to $L^{\prime}$ is the tree with leaf set $L^{\prime}$ obtained from $T_{\text {lcaT }\left(L^{\prime}\right)}$ by removing all leaves that are not in $L^{\prime}$, and all internal nodes of degree 2, except the root. Let $T^{\prime}$ be a tree such that $L\left(T^{\prime}\right)=L^{\prime} \subset L(T)$. We say that $T$ displays $T^{\prime}$ iff $\left.T\right|_{L^{\prime}}$ is label-isomorphic to $T^{\prime}$.

A triplet is a binary tree on a set $L$ with $|L|=3$. For $L=\{x, y, z\}$, we denote by $x y \mid z$ the unique triplet $t$ on $L$ with root $r(t)$ for which $l c a_{t}(x, y) \neq r(t)$ holds. We denote by $\operatorname{tr}(T)=\left\{\left.T\right|_{L}: L \in\left(\begin{array}{c}L(T) \\ 3\end{array}\right)\right.$ and $\left.T\right|_{L}$ is binary $\}$ the set of all rooted triplets of a tree $T$.

Evolution of species and genes: A species tree $S$ for $\Sigma$ is a rooted tree whose leaves are in bijection with $\Sigma$, representing the evolutionary relationships between the species: an internal node is an ancestral species at the moment of a speciation event, and its children are the descendants. Although species trees are generally binary, we do not make this assumption here. Genes of $\mathcal{G}$ undergo speciation when the species to which they belong do. Within a species, genes can be duplicated or lost. A history $H$ for $\mathcal{G}$ is a tree representing the evolution of the gene family through speciations and duplications: each leaf of $H$ is labeled by a 
gene of $\mathcal{G}$, and each internal node refers to an ancestral gene at the moment of an event (speciation or duplication). Therefore each internal node of $H$ can be labeled as a speciation $(\mathrm{Spec})$ or duplication (Dup) event.

As $H$ is a history "embedded" in the species tree $S$ of $\Sigma$, it must reflect a speciation history consistent with $S$ : any speciation node of $H$ should reflect a clustering of species in agreement with $S$. To formally define consistency, let first introduce a more general set of labeled trees. We call a $D S$-tree for $\mathcal{G}$ a pair $(G, \ell)$, where $G$ is a tree with $L(G)=\mathcal{G}$, and $\ell$ is a function $\ell: V(G) \backslash L$ $(G) \rightarrow\{$ Dup, Spec $\}$ labeling each internal node of $G$ as a duplication or a speciation node. For simplicity, we often refer to $G$ as the $D S$-tree for $\mathcal{G}$ without explicitly stating $\ell$, and assume the internal nodes of $G$ are labeled Dup or Spec. For some $X \subseteq L(G)$, we implicitly assume that the internal nodes of $\left.G\right|_{X}$ share the same label as in $G$.

Definition 1 Let $G$ be a DS-tree for $\mathcal{G}$ and $S$ be a species tree for $\Sigma$. We say that $G$ is consistent with $S$ if and only if, for any speciation node $x$ of $G$ and any two children $y, z$ of $x, l c a_{S}\left(s\left(L_{y}\right)\right)$ and $l c a_{S}\left(s\left(L_{z}\right)\right)$ are unrelated in $S$, where $L_{y}$ and $L_{z}$ are the leaf-sets of $G_{y}$ and $G_{z}$ respectively.

Now a history $H$ for $\mathcal{G}$ is simply a $D S$-tree for $\mathcal{G}$ consistent with the species tree $S$ for $\Sigma$. Denote

$\operatorname{tr}_{S}(G)=\{s(x) s(y)|s(z): x y| z \in \operatorname{tr}(G)$ is rooted at a speciation and $s(x) \neq s(y)\}$

The triplets of $\operatorname{tr}_{S}(G)$ are called speciation triplets. The following theorem, which is a reformulation of Theorem 6 in [18], relates consistency to speciation triplets.

Theorem 1 Let $G$ be a DS-tree for $\mathcal{G}$ and $S$ be a species tree for $\Sigma$. Then $G$ is consistent with $S$ if and only if $S$ displays all triplets of $\operatorname{tr}_{S}(G)$.

From the Fitch [20] terminology, two leaves $x$ and $y$ of a history are orthologous if $l c a_{H}(x, y)$ is a speciation node, and paralogous otherwise. We extend this terminology to a more general $D S$-tree.

Definition 2 Let $G$ be a DS-tree for $\mathcal{G}$. Then two genes $x, y$ of $G$ are orthologous with respect to (w.r.t.) $G$ if $l c a_{G}$ $(x, y)$ is a speciation node, and paralogous w.r.t. $G$ if $l c a_{G}(x, y)$ is a duplication node.

Therefore a $D S$-tree induces a set of orthology/paralogy relationships between genes.

Constraint graph: A constraint is simply an unordered pair of genes $\{x, y\} \in\left(\begin{array}{l}\mathcal{G} \\ 2\end{array}\right)$. A set of orthology/paralogy constraints on $\mathcal{G}$ (or simply a constraint set) is a pair $\mathrm{C}=$ $\left(C_{O}, C_{P}\right)$ of subsets $C_{O}, C_{P} \subset\left(\begin{array}{l}\mathcal{G} \\ 2\end{array}\right)$ such that $C_{O} \cap C_{P}=\varnothing$. $C_{O}$ represents the orthology constraints and $C_{P}$ the paralogy constraints. We say that $C$ is a full constraint set if
$\mathcal{G}$. For example, a history $H$ for $\mathcal{G}$ induces a full constraint set.

We represent a constraint set $C=\left(C_{O}, C_{P}\right)$ by an edgebicoloured undirected graph $R=(V, E, U)$, called a constraint graph, with vertex set $V=\mathcal{G}$, and two edge sets $E=C_{O}$ and $U=\left(\begin{array}{l}\mathcal{G} \\ 2\end{array}\right) \backslash\left(C_{O} \cup C_{P}\right)$. Said differently, two genes are linked by an edge of $E$ if they are constrained by orthology, unlinked if they are constrained by paralogy, and linked by a "special" edge of $U$ if the relation between the two genes is unknown. We refer to the edges of $E$ as the orthology edges, to those in $U$ as the unknown edges and we refer to the unlinked pairs of genes as the paralogy non-edges. An example of a partial constraint graph is given in Figure 1.

If $C$ is a full constraint set then $U=\emptyset . R$ is called a full constraint graph in this case. The complement of $R$ is the graph $\bar{R}$ obtained by the alternative choice of linking paralogs instead of orthologs. Formally, $\bar{R}=(V, \bar{E} \backslash U, U)$, where $\bar{E}$ is the complement of $E$ defined by $e \in \bar{E}$ iff $e \notin E$. Notice that $R$ and $\bar{R}$ share the same set $U$ of unknown edges. We denote by $R[X]$ the graph $R$ induced by $X \subseteq V$, i.e. $R[X]=(X, E(X), U(X))$ where $E(X)$ (resp. $U(X))$ are the edges of $E$ (resp. $U$ ) having both endpoints in $X$. Note that if $U=\emptyset, R[X]$ corresponds to the usual definition of the graph induced by $X$.

Satisfiability and consistency Given a constraint set $C$ (or similarly a constraint graph $R$ ), is $C$ possible, i.e. can we find a history for $\mathcal{G}$ inducing the orthology and paralogy constraints of $C$ ? As an orthology constraint for two genes belonging to the same genome cannot be induced by a history for $\mathcal{G}$, we assume in the rest of this paper that the set $\tilde{C}_{P}=\left\{\{x, y\} \in\left(\begin{array}{l}\mathcal{G} \\ 2\end{array}\right): s(x)=s(y)\right\}$ is included in $C_{P}$. A trivial set of paralogy constraints is a set $C_{P}$ restricted to $\tilde{C}_{P}$.

The question whether $C$ is possible is in two parts: (1) is $C$ satisfiable, i.e. can we find a $D S$-tree $G$ inducing $\mathcal{C}$ and (2) is there such a $G$ which is consistent with a species tree? Formal definitions follow.

Definition 3 Let $R=(V, E, U)$ be a constraint graph and $G$ be a DS-tree with $L(G)=V$. We say that $G$ satisfies $R$ if for two genes $x, y \in L(G)$, if $x y \in E$ then they are orthologous w.r.t. $G$, and if $x y \in E \backslash U$ then they are paralogous w.r.t. G. We say that $R$ is satisfiable if there exist a $D S$-tree $G$ that satisfies $R$.

If $U=\emptyset$, then $R$ being satisfiable means that we can make a choice for the unknown edges as orthology edges and paralogy non-edges to obtain a full constraint graph that is satisfiable. For $F \subset U$, the realization of $R$ by $F$ corresponds to choosing $F$ as orthology edges, and $U \backslash F$ as paralogy non-edges, leading to the full constraint 


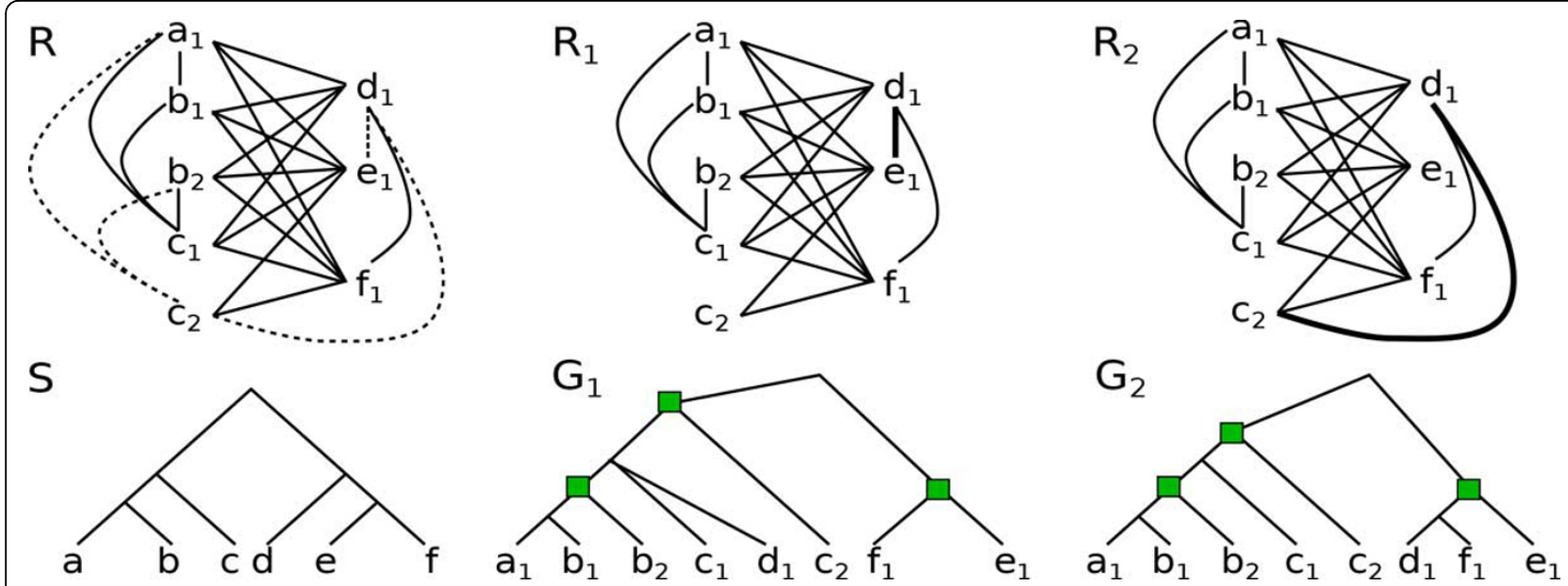

Figure 1 Constraint graph, satisfiability and consistency. A constraint graph $R=(V, E, U)$ with $E$ and $U$ depicted by solid and dotted edges, along with with two satisfiable realizations $R_{1}=R\left(\left\{d_{1} e_{1}\right\}\right)$ and $R_{2}=R\left(\left\{d_{1} c_{2}\right\}\right)$. The gene names correspond to their respective species in the species tree $S$. The tree $G_{1}$ is a DS-tree that satisfies $R_{1}$ while $G_{2}$ is a DS-tree that satisfies $R_{2}$. Duplication nodes in $G_{1}$ and $G_{2}$ are indicated by a green square. $G_{1}$ is not consistent with $S$ because for instance, $G_{1}$ has the speciation triplet $s\left(a_{1}\right) s\left(d_{1}\right)\left|s\left(e_{1}\right)=a d\right| e$ while $S$ has the de|a triplet. The tree $G_{2}$ is consistent with $S$.

graph $R(F)=(V, E \cup F, \varnothing)$. We call $R(\not)$ and $R(U)$ the empty and full realizations, respectively.

As a history is a $D S$-tree, a set of constraints that is not satisfiable is clearly not possible, i.e. there is no history that depicts the orthology/paralogy relationships given by the constraints. Moreover, satisfiability is not sufficient to ensure the possibility of such an history, as a $D S$-tree is not always consistent with a species tree. Figure 1 shows an example of a constraint graph $R$ along with two satisfying realizations $R_{1}$ and $R_{2}$. However, $R_{1}$ cannot be made consistent with a given species $S$ whereas $R_{2}$ can.

Definition 4 Let $R$ be a constraint graph for $\mathcal{G}$. We say that $R$ is consistent with a species tree $S$ if and only if there is a realization of $R$ which is satisfiable by a DStree $G$ which is consistent with S. More generally, we say that $R$ is consistent if and only if there is a species tree $S$ such that $R$ is consistent with $S$.

The three following sections are respectively dedicated to the three following questions: (1) Given a constraint graph $R=(V, E, U)$, is $R$ satisfiable? (2) Given a satisfiable constraint graph $R=(V, E, U)$, and a species tree $S$, is $R$ consistent with $S$ ? (3) Given a satisfiable constraint graph $R=(V, E, U)$, is $R$ consistent, i.e. can we find a species tree $S$ such that $R$ is consistent with $S$ ?

\section{Satisfiability of a constraint graph}

The problem of constraint graph satisfiability has been addressed in [17] in the restricted case of a full set of constraints. The following theorem is a reformulation of one of the main results of this paper.
Theorem 2 ([17]) A full constraint graph $R$ is satisfiable if and only if $R$ is $P_{4^{-}}$free (or equivalently, iff $\bar{R}$ is $P_{4}$-free since $P_{4}$ is self-complementary), meaning that no four vertices of $R$ induce a path of length 4.

Consider now the general case of a constraint graph $R=(V, E, U)$ with $U=\emptyset$. Then the problem is to find a realization $R(F)$ that is itself satisfiable, i.e. $P_{4}$ - free. It turns out that this problem is a reformulation of the well-known Graph sandwich problem for $P_{4}$-free graphs. It can be stated as follows : given two graphs $G_{1}=\left(V, E_{1}\right)$ and $G_{2}=\left(V, E_{2}\right)$, with $E_{1} \subseteq E_{2}$, does there exist a $P_{4}$-free graph $G=(V, E)$ such that $E_{1} \subseteq E \subseteq E_{2}$. That is, G must contain every edge of $G_{1}$ and every non-edge of $G_{2}$. It is then clear that this is equivalent to finding a $P_{4}$-free realization of $R=\left(V, E_{1}, U=E_{2} \backslash E_{1}\right)$. A $O\left(|V|^{3}\right)$ algorithm was proposed in [21] to solve this problem. In this section, we restate under our formalism some of the useful results of this paper, and give a modified version of the proposed algorithm that outputs a $D S$ tree that satisfies $R$ whenever there is one. We will make use of the following well-known characterization of $P_{4}$-free graphs.

Lemma $1 A$ graph $\Gamma$ is $P_{4}$-free if and only if, for any subset $X$ of vertices of $\Gamma$ with $|X| \geq 2$, either $\Gamma[X]$ or $\overline{\Gamma[X]}$ is disconnected.

The next lemmata establish an important heritability property on satisfiable graphs: every restriction $R[X]$ of $R$ must be satisfiable for $R$ to be satisfiable.

Lemma 2 Let $G$ be a DS-tree that satisfies a realization $R(F)$, for some $F \subseteq U$. Let $X \subseteq V$ and let $F_{X}=\{a b \in$ $F: a, b \in X\}$. Then $\left.G\right|_{X}$ is a realization of $R[X]\left(F_{X}\right)$. 
Proof: Let $a, b \in X$. First observe that $a$ and $b$ have the same orthology/paralogy relationship in $R(F)$ and $R[X]$ $\left(F_{X}\right)$. Let $c=l c a_{G}(a, b)$. Now, $c$ is also an internal node of $\left.G\right|_{X}$, and moreover $c=l c a_{G \mid X}(a, b)$. As $c$ has the same Dup or Spec label as in G, $c$ correctly displays the relationship between $a$ and $b$. Thus $\left.G\right|_{X}$ satisfies every relationship in $R[X]\left(F_{X}\right)$.

The heritability property is then immediate.

Lemma 3 [21]A constraint graph $R=(V, E, U)$ is satisfiable if and only if for any $X \subseteq V, R[X]$ is satisfiable.

Theorem 3 [21]A constraint graph $R$ is satisfiable if and only if at least one of the two following holds :

$1 R(\not)$ is disconnected, and all of its connected components are satisfiable;

$2 \overline{R(U)}$ is disconnected, and all of its connected components are satisfiable.

Proof: $\Leftarrow$ For 1.: Suppose $R(\not)$ is disconnected. Let $\left\{R_{1}, \ldots, R_{k}\right\}$ be the connected components of $R(\not)$ with $k>1$, all being satisfiable. As each $R_{i}$ is satisfiable, there is a $D S$-tree $G_{i}$ that satisfies a realization $R_{i}\left(F_{i}\right)$ of $R_{i}$. Let $F=U_{1 \leq i \leq k} F_{i}$. Then the realization $R(F)$ of $R$ is a full constraint graph with $k$ full constraint components $R_{i}$ $\left(F_{i}\right)$ in which no two components share an edge. In other words, there is a paralogy non-edge between each pair of genes from two different components. Therefore, joining the roots of $G_{1}, \ldots, G_{k}$ under a common duplication node yields a $D S$-tree for $R(F)$, which shows that $R$ is satisfiable. The proof when 2 . holds is the same, except that we root $\mathrm{G}$ at a speciation node since the components of $\overline{R(U)}$ are pairwise-complete in $R(U)$.

$\Rightarrow$ Suppose that both conditions do not hold. If $R(\not)$ or $\overline{R(U)}$ has a component that is not satisfiable, then by Lemma $3, R$ is not satisfiable. So instead suppose that each of $R(\varnothing)$ and $\overline{R(U)}$ has a single connected component. Let $F \subseteq U$. The realization $R(F)$ of $R$ must be connected as $R(\not)$ is already connected and $\overline{R(F)} \cdot \overline{R(F)}$ must also be connected, as choosing all edges of $U$ leaves $\overline{R(U)}$ connected and $E \overline{R(U)} \subseteq E \overline{(R(E))}$. Since both $R(F)$ and $\overline{R(F)}$ are connected, by Lemma $1 R$ $(F)$ is not $P_{4}$-free, and thus not satisfiable by Theorem 2 . As this is true for any realization $R(F)$ of $R$, i.e. for any $F \subseteq U$, it follows that $R$ is not satisfiable.

Theorem 3 suggests the recursive algorithm BuildDSTree that begins by finding out if one of $R(\varnothing)$ or $\overline{R(U)}$ is disconnected. If so, it creates a node of the appropriate type with children being the identified components, and repeats the process on each such component.

Algorithm BuildDSTree $(R=(V, E, U), v)$

where $R$ is a (possibly induced) constraint graph and $v$ is the current node of $G$ we are creating

$$
\operatorname{IF}|V|=1 ; \operatorname{RETURN} ; R(\not)=(V, E, \varnothing)
$$

Find the connected components $C C$ of $R(\not)$ through a depth-first search

$$
\begin{aligned}
\text { IF }|C C| & >1 ; \\
\text { type } & \leftarrow \text { Dup }
\end{aligned}
$$

ELSE

$\overline{R(U)}=(V, \bar{E}, \emptyset)$

type $\leftarrow$ Spec

Find the connected components $C C$ of $\overline{R(U)}$

through a depth-first search IF $|C C|=1$; output

"Unsatisfiable", and halt the recursion

\section{END IF}

v.type $\leftarrow$ type

FOR $C \in C C$;

Add child node $v_{C}$ to $v$

BuildDST ree $(\mathrm{R}[\mathrm{C}], \mathrm{vC})$

END FOR

RETURN

If $n$ is the number of genes in $G$, the algorithm creates a $D S$-tree $G$ with at most $n-1$ internal nodes (or stops before if $R$ is unsatisfiable). For each such internal node $v$, the time taken to go through the algorithm is dominated by (at most) two depth-first searches that are performed on $L\left(G_{v}\right)$, and the rest of the work is handled by children nodes. So the time taken to handle $v$ is bounded by the number of edges/non-edges in $R\left[L\left(G_{v}\right)\right]$, which is $O\left(\left|L\left(G_{v}\right)\right|^{2}\right) \subseteq O\left(n^{2}\right)$. Therefore the timecomplexity of BuildDSTree is in $O\left(n^{3}\right)$.

\section{Consistency with a given species tree}

Let $R=(V, E, U)$ be a constraint graph for $\mathcal{G}$ and $S$ be a species tree for $\Sigma$. We want to know whether the orthology/paralogy constraints represented by $R$ can be induced by a history for $\mathcal{G}$ consistent with $S$. More precisely, is there a realization $R(F)$ of $R$ that is satisfiable and such that the DS-tree satisfying $R(F)$ is consistent with $S$ ? If $R$ is not satisfiable, then the answer is clearly no. Therefore hereafter we assume that $R$ is satisfiable. We first show that the problem at hand still has the heritability property.

Lemma $4 R$ is consistent with $S$ if and only if for any $X \subseteq V, R[X]$ is consistent with $S$.

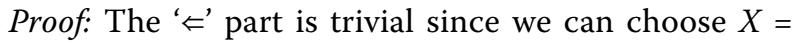
$V$ to show that $R$ is consistent with $S$. Conversely, assume $R$ is consistent with $S$. Let G be a $D S$-tree for some realization of $R$ such that $\mathrm{G}$ is consistent with $S$, and let $X \subseteq V$. By Lemma $2,\left.G\right|_{X}$ is a $D S$-tree for $R[X]$. Let $a b \mid c \in \operatorname{tr}_{S}\left(\left.G\right|_{X}\right)$. Since going from $\left.G\right|_{X}$ to $G$ only involves adding subtrees on branches of $\left.G\right|_{X}$, it follows that $a b \mid c \in \operatorname{tr}_{S}(G)$. Therefore, $\operatorname{tr}_{S}\left(\left.G\right|_{X}\right) \subseteq \operatorname{tr}_{S}(G)$. Now, since $S$ displays $\operatorname{tr}_{S}(G),\left.G\right|_{X}$ is a realization of $R[X]$ that is consistent with $S$.

We need to introduce one last notation before stating the main theorem for characterizing consistency of a 
constraint graph $R$ with a species tree $S$. Let $R(F)$ be a realization of $R$, and let $C C=\left\{R_{1}, \ldots, R_{k}\right\}$ be the connected components of $\overline{R(F)}$. Notice that the components of $C C$ are pairwise complete in $R(F)$. A speciation partition $P=\left\{P_{1}, \ldots, P_{|P|}\right\}$ is a non-trivial partition of $C C$ (i.e. $\mid$ $P \mid>1)$ such that $l c a_{S}\left(s\left(P_{i}\right)\right)$ is unrelated to $l c a_{S}\left(s\left(P_{j}\right)\right)$ whenever $i \neq j$.

Theorem $4 R$ is consistent with $S$ if and only if at least one of the following conditions holds:

$1 R(\varnothing)$ is disconnected and each connected component is consistent with $S$;

$2 \overline{R(U)}$ is disconnected, its components admit a speciation partition and each component in this partition is consistent with $S$.

Proof: $\Leftarrow$ For 1 ., Let $\left\{R_{1}, \ldots, R_{k}\right\}$ be the connected components of $R(\varnothing)$, each $R_{i}$ having a $D S$-tree $G_{i}$ consistent with $S$. We can then join the roots of $G_{1}, \ldots, G_{k}$ under a common duplication parent. This yields a $D S$-tree $\mathrm{G}$ that satisfies $R$ as each pair of components of $R(\not)$ are related by paralogy. Furthermore, all rooted triplets of $G$ that were not in any $G_{i}$ are rooted at $r(G)$, a Dup node. Therefore, $\operatorname{tr}_{S}(G)=U_{1 \leq i \leq k} \operatorname{tr}_{S}\left(G_{i}\right)$, which $S$ displays.

$\Leftarrow 2$ : Let $P=\left\{P_{1}, \ldots, P_{k}\right\}$ be a non-trivial speciation partition of the connected components of $\overline{R(U)}$. By assumption every $P_{i} \in P$ has a $D S$-tree $G_{i}$ that is consistent with $S$, implying that $S$ displays $\cup_{1 \leq i \leq k} \operatorname{tr}_{S}\left(G_{i}\right)$. Here all elements of $P$ are components of $R$ that are pairwisecomplete, and we obtain a $D S$-tree $\mathrm{G}$ for $R$ by joining $G_{1}, \ldots, G_{k}$ under a common speciation parent. Let $T=t_{S}$ (G) $\backslash \mathrm{U}_{1 \leq i \leq k} \operatorname{tr}_{S}\left(G_{i}\right)$. Every triplet of $T$ is rooted at $r(G)$. Thus if three genes $a, b, c$ of $L(G)$ form a speciation triplet $s(a) s(b) \mid s(c) \in T$, then $a$ and $b$ are in some part $P_{i}$ while $c$ is in another part $P_{j}$. But by the definition of speciation partitions, $l c a_{S}\left(s\left(P_{i}\right)\right)$ is unrelated to $l c a_{S}\left(s\left(P_{j}\right)\right)$, implying that $s(a) s(b) \mid s(c) \in \operatorname{tr}(S)$. It follows that $S$ displays $T$.

$\Rightarrow$ : suppose both conditions are not met, but that $R$ is consistent with $S$. If $R(\varnothing)$ is disconnected but has an inconsistent component, then $R$ is inconsistent by Lemma 4. So we assume $R(\varnothing)$ is connected. If $\overline{R(U)}$ is also connected, then we saw in Theorem 3 that $R$ is not even satisfiable. If $\overline{R(U)}$ is disconnected and admits a speciation partition, but a member of this partition is not consistent, then again by Lemma $4, R$ is not consistent. So we assume that $R(\not)$ is connected, and $\overline{R(U)}$ is disconnected but admits no speciation partition. Let $G$ be a $D S$-tree for $R$ consistent with $S$. Suppose $r(G)$ is a duplication node and let $r_{1}, r_{2}$ be two children of $r(G)$. We have that every gene in $L\left(G_{r_{1}}\right)$ is paralogous with every gene in $L\left(G_{r_{2}}\right)$ and vice-versa. This implies that $L$ $\left(G_{r_{1}}\right)$ and $L\left(G_{r_{2}}\right)$ are two components of $R(\not)$ that share no edge, a contradiction since we assume $R(\not)$ is connected. So $r(G)$ is a speciation node. Let $r_{1}, \ldots, r_{k}$ be the children of $r(G)$. The sets $L\left(G_{r_{1}}\right), \ldots, L\left(G_{r_{\mathrm{k}}}\right)$ form a partition $P$ of the connected components of $\overline{R(U)}$. Since $S$ displays $\operatorname{tr}_{S}(G)$, it follows that for two distinct $P_{i}, P_{j} \in$ $P$, $l c a_{S}\left(s\left(P_{i}\right)\right)$ and $l c a_{S}\left(s\left(P_{j}\right)\right)$ are unrelated.

Hence $P$ is a speciation partition, a contradiction.

This theorem suggests a small modification to algorithm BuildDSTree. Connected components of $R(\not)$ are handled in the same manner, but in the case of a disconnected $\overline{R(U)}$, we need to find a speciation partition $P$ after having found its connected components $C C$. To accomplish this, it suffices to observe that some $C_{1}, C_{2} \in C C$ must be in the same part of $P$ when $l c a_{S}\left(s\left(C_{1}\right)\right)$ is on the path from $l c a_{S}\left(s\left(C_{2}\right)\right)$ to the root of $S$ (or vice-versa). Thus for each $P_{i} \in P$, we can find the member of $C \in P_{i}$ that has $l c a_{S}(s(C))$ the closest to the root of $S$, then any other component $C^{\prime}$ having $l c a_{S}\left(s\left(C^{\prime}\right)\right)$ in the subtree rooted at $l c a_{S}(s(C))$ will be in $P_{i}$.

FINDSPECIATIONPARTITION uses that fact to find $P$ through a pre-order traversal of $S$.

Algorithm FindSpeciationPartition $(C C, s, P, P i)$

where $C C$ is the set of components to partition, $s \in V$ $(S)$ is the current node of $S$ in the pre-order traversal, $P$ is the partition of $C C$, and $P i$ is the current part of $P$ we are adding components to

FOR $C \in C C$ such that $l c a_{S}(C)=s$;

IF $P_{i}$ is not set; let $P_{i}$ be a new empty set and add $P_{i}$ to $P$

Add $C$ to $P_{i}$

END FOR

FOR $\mathrm{s}^{\prime} \in$ children(s);

FindSpeciationPartition $\left(C C, s^{\prime}, P, P_{i}\right)$

\section{END FOR}

Assuming constant time for each lca lookup, we can precompute $l c a_{S}(s(C))$ in time $|C|$ for each $C \in C C$. If $C C$ has a total of $k$ nodes, by mapping each $s \in S$ to the list of $C \in C C$ with $l c a_{S}(s(C))=s$, the whole algorithm takes time $O(k+|S|)$. We need up to $n-1$ calls of BuildDSTree. We argued that one call on a node $v$ of $G$ in BUILDDSTREE takes time $O\left(\left|L\left(G_{v}\right)\right|^{2}\right)$, so adding this step makes it $O\left(\left|L\left(G_{v}\right)\right|^{2}+|S|+k\right)$. Noting that $k=\left|L\left(G_{v}\right)\right|$, and assuming that $\left|L\left(G_{v}\right)\right| \geq|S|$, this modified algorithm still runs in time $O\left(n^{3}\right)$, where $n=|\mathcal{G}|$.

\section{Consistency of a satisfiable constraint graph}

Now let $R=(V, E, U)$ be a constraint graph for $\mathcal{G}$ and suppose the species tree for $\Sigma$ is unknown. The question is to know whether the graph $R$ is consistent, and if so to output a species tree $S$ such that $R$ is consistent with $S$. As above, we assume that $R$ is satisfiable. Note that unlike the two previous problems, we cannot treat each connected component of $R(\not)$ or $\overline{R(U)}$ independently, as two (or more) components might give gene histories consistent by themselves but not together. 
We hereafer begin with two classes of constraint graphs for which consistency always holds.

Orthology constraints only: Suppose $R=(V=\mathcal{G}, E, U)$ represents a constraint set restricted to orthology constraints, i.e. $C=\left(C_{O}, C_{P}\right)$, where $C_{P}=\tilde{C}$ is the trivial set of paralogy constraints. For each $s_{i} \in \Sigma$, let $L_{s_{i}}=\left\{x \in V: s(x)=s_{i}\right\}$ and let $F_{s_{i}}$ be the star-tree joining all the leaves of $L_{s_{i}}$ under a single duplication node. Now let $G$ be the star-tree joining all the $F_{s_{i}}$ trees, for all $s_{i} \in \Sigma$, under a single speciation node. Then $G$ satisfies the full realization $R(U)$ of $R$ and it is consistent with the star-tree for $\Sigma$. Therefore any set of orthology constraints is consistent.

Paralogy constraints only: Suppose $R=(V=\mathcal{G}, E, U)$ represents a constraint set restricted to paralogy constraints, i.e. $C=\left(C_{O}=\emptyset, C_{P}\right)$. If $G$ is the star-tree joining all the genes of $\mathcal{G}$ under a single duplication node, then $G$ satisfies the empty realization $R(\varnothing)$ of $R$ and it is consistent with the star-tree for $\Sigma$. Therefore any set of paralogy constraints is consistent.

Consider now a full constraint graph $R$. The results in $[17,18]$ suggest a polynomial-time algorithm for solving the consistency problem that consists in building a $D S$ tree $G$ satisfying $R$, extracting all speciation triplets of $G$ and checking their consistency with a species tree. Here we propose an alternative polynomial time algorithm for the same problem, avoiding the first step of a $D S$-tree construction. We first introduce the following subset $P_{3}$ $(R)$ of triplets of $\left(\begin{array}{l}V \\ 3\end{array}\right)$ inducing a path of size 3 in $R$ :

$$
P_{3}(R)=\left\{s(x) s(y) \mid s(z):\{x, y, z\} \in\left(\begin{array}{l}
V \\
3
\end{array}\right), z x, z y \in E \text { and } x y \notin E \cup U \text { and } s(x) \notin s(y)\right\}
$$

Notice that $s(x) s(y) \mid s(z) \in P_{3}(R)$ implies that any $D S$-tree $G$ satisfying $R$ has $s(x) s(y) \mid s(z) \in \operatorname{tr}_{S}(G)$. Indeed, since $x y \notin E$ $\cup U, l c a_{G}(x, y)$ is a duplication node. And since both $x$ and $y$ are related to $z$ by speciation, $l c a_{G}(x, z)=l c a_{G}(y, z)$ and $x y \mid z$ must be a speciation triplet of $G$.

For example, consider the vertices $b_{1}, c_{2}, e_{1}$ of the $R$ graph in Figure 1, which form a path of length 3 with $e_{1}$ in the center. In the $D S$-tree $G_{1}, l c a_{G_{1}}\left(b_{1}, c_{2}\right)$ is a duplication, and $l c a_{G_{1}}\left(b_{1}, c_{2}\right)\left(\left\{b_{1}, c_{2}, e_{1}\right\}\right)$ is a speciation. Restricting $G_{1}$ to the three vertices yields the triplet $b_{1} c_{2} \mid e_{1}$ rooted at a speciation, and therefore, $s\left(b_{1}\right) s\left(c_{2}\right) \mid s\left(e_{1}\right) \in \operatorname{tr}_{S}\left(G_{1}\right)$.

The same holds for the $s\left(c_{1}\right) s\left(c_{2}\right) \mid s\left(e_{1}\right)$ triplet implied by the $P_{3}$ induced by $c_{1}, c_{2}, e_{1}$. Notice however that in both $D S$-trees, $s\left(b_{1}\right) s\left(c_{1}\right) \mid s\left(e_{1}\right)$ is a speciation triplet, though $b_{1}$, $c_{1}, e_{1}$ do not induce a $P_{3}$. We show that this kind of speciation triplet is implied by the other two aforementioned $P_{3}$, and that the $P_{3}$ subgraphs actually imply every mandatory speciation triplet.

Theorem 5 Let $R=(V, E, U=\emptyset)$ be a satisfiable full constraint graph. Then $R$ is consistent if and only if there exists a species tree $S$ displaying all the triplets of $P_{3}(R)$.
Proof: $\Rightarrow$ : since $s(x) s(y) \mid s(z) \in P_{3}(R)$ implies that $s(x) s$ $(y) \mid s(z) \in \operatorname{tr}_{S}(G)$, it follows that any species tree $S$ consistent with $R$ must display every triplet of $P_{3}(R)$.

$\varepsilon$ : we first obtain a least-resolved $D S$-tree $G$ for $R$ in terms of speciation. Let $G^{\prime}$ be a consistent $D S$-tree satisfying $R$, and let $S$ be a species tree displaying $P_{3}$ $(R)$. If $G^{\prime}$ has any speciation node $v$ that has a speciation child $w$, we obtain $G^{\prime \prime}$ by contracting $v$ and $w$ (delete $w$ and give its children to $v$ ). Since $v$ and $w$ are both speciations, this operation does not change the label of $l c a_{G}(x, y)$ for any two leaves $x$ and $y$ and $G^{\prime \prime}$ still satisfies $R$. Moreover, $\operatorname{tr}_{S}\left(G^{\prime \prime}\right) \subset \operatorname{tr}_{S}\left(G^{\prime}\right)$, so there is no risk of breaking consistency. We obtain a $D S$-tree $G$ by repeating this operation until we cannot find such a $v$ and $w$.

Let $x y \mid z$ be a triplet of $G$ rooted at a speciation node. We have that $l c a_{G}(z, x)=l c a_{G}(z, y)$ is a speciation, and that $z x, z y \in E$. If $l c a_{G}(x, y)$ is a duplication node, then $x y \notin E$. So $\{x, y, z\}$ induces a $P_{3}$ in $R$, and $S$ displays $s(x)$ $s(y) \mid s(z)$. Suppose instead that $l c a_{G}(x, y)$ is a speciation node. Because $G$ is a least resolved $D S$-tree, there must be a duplication node $u$ on the path between $l c a_{G}(x, y)$ and $l c a_{G}(x, z)$. This implies there is a leaf $d$ in $G_{u}$ such that $x$ and $y$ are related to $d$ by duplication, but with $d$ and $z$ related by speciation. In $R$, we then have $z d \in E$, and $x d, y d \notin E$. Thus both $\{x, d, z\}$ and $\{y, d, z\}$ induce a $P_{3}$ in $R$ with $z$ being the middle vertex, and $s(x) s(d) \mid s(z)$, $s(y) s(d) \mid s(z) \in P_{3}(R)$ are both displayed by $S$. This is only possible if there is a node in $S$ that has all of $s(x), s(y), s$ $(d)$ in one child subtree and $s(z)$ in another. Therefore, $S$ must display $s(x) s(y) \mid s(z)$. Having taken care of both types of speciation triplets, we deduce that displaying $P_{3}$ $(R)$ is sufficient to display $\operatorname{tr}_{S}(G)$.

Therefore the consistency problem for a satisfiable full constraint graph reduces to the problem of verifying whether the set $P_{3}(R)$ of triples can be displayed in a species tree for $\Sigma$. This is in fact a well know problem with a solution presented in [22]: given a triplet set $\mathcal{R}$, there is a polynomial-time algorithm, called BUILD [23], that, when applied to $\mathcal{R}$ either outputs a species tree that displays $\mathcal{R}$ or recognizes that $\mathcal{R}$ is inconsistent. Therefore, in the case of a full constraint graph, the consistency problem is resolved in polynomial time by first constructing the set $P_{3}(R)$, and then applying the BUILD algorithm.

Consider now the general case of a constraint graph $R=(V, E, U)$ with $U \neq 0$. The branch-and-bound algorithm CHECKCONS iterates over the edges of $U$, tries to make them edges and non-edges but stops as soon as one decision creates a set of $P_{4}$ with no unknowns or a set of $P_{3}$ that is inconsistent. Since at worst, every possibility is tested, it follows that this algorithm is exact, though exponential in the worst case. 


\section{ALGORITHM CHECKCONS $(R=(V, E, U))$}

Obtain a species tree $S$ by running BUILD on $P_{3}(R)$ IF $S$ is not set (i.e. BUILD failed), RETURN FALSE IF $R$ is not satisfiable, RETURN FALSE

IF $U=\emptyset$, return $(R, S)$

Let $\mathrm{e} \in \mathrm{U}$ and let $\mathrm{R}_{\mathrm{e}}=(\mathrm{V}, \mathrm{E} \mathrm{U}\{\mathrm{e}\}, \mathrm{U} \backslash\{\mathrm{e}\})$

$\left(\mathrm{R}^{\prime}, \mathrm{S}\right) \leftarrow$ CHECKCON $\mathrm{S}\left(\mathrm{R}_{\mathrm{e}}\right)$

IF $\left(R^{\prime}, S\right)$ is set (i.e. CHECKCONS succeeded), RETURN $\left(R^{\prime}, S\right)$ Otherwise let $R_{\bar{e}}=(V, E, U \backslash\{e\})$

$\left(\mathrm{R}^{\prime}, \mathrm{S}\right) \leftarrow \operatorname{CHECKCONS}\left(R_{\bar{e}}\right)$

IF $\left(R^{\prime}, S\right)$ is set (i.e. CHECKCONS succeeded), RETURN $\left(R^{\prime}, S\right)$

Otherwise RETURN FALSE

Possible improvements of this algorthm include removing as many edges from $U$ as possible, and choosing an ordering of the edges that may speed up the branch-and- bound process. For instance, it may be worthwhile to first identify every induced $P_{4}$ of $R(\not)$. The $P_{4}$ subgraphs that admit only one possibility for removal, i.e. the $P_{4}$ can only be removed by making a unique edge $e \in U$ an orthology edge, can be corrected before entering the algorithm. Note that the same applies for the edges of $U$ that must be edges of $\overline{R(U)}$. We may then prioritize the handling of the other $P_{4}$ by considering the edges that resolve them first. Similarly, it would also be possible to identify edges of $U$ that are mandatory in $E$ by finding the $P_{3}$ subgraphs of $R(\varnothing)$ that are not in $P_{3}(R)$, but that disagree with a triplet of $P_{3}(R)$. For instance, $R(\varnothing)$ might have a $P_{3}$ with edges $x z$, $z y$, but this $P_{3}$ is not in $P_{3}(R)$ because $x y \in U$. If say $s(y) s(z) \mid s(x)$ is in $P_{3}(R)$, then $x y$ is forced in $E$ as otherwise the contradictory $s(x) s(y) \mid s(z)$ triplet would be present.

\section{Experiments}

We show how the developed algorithms for checking satisfiability and consistency can be used, in combination with an orthology detection tool such as ProteinOrtho [11], to infer a robust set of orthology and paralogy constraints. Given a set of protein sequences, Proteinortho infers homologous gene families as well as orthology relationships within these families, based on various similarity scores. Proteinortho does not infer paralogy relationships. However, if we choose a set of parameters leading to a loose characterization of orthologs, then we can assume that unpredicted constraints should represent paralogy. Different combinations of parameters therefore lead to different constraint sets that can be analyzed for sat-isfiability and consistency.

ProteinOrtho has been run on 265 gene families of vertebrates, each representing the leaf-set of an Ensembl [5] gene tree. Trees were chosen randomly among the Ensembl gene trees containing at least 20 leaves. For each family, five different parameter settings, numbered from -2 to +2 , were tested, 0 representing the default parameter choice of ProteinOrtho, and the smaller the number, the looser is the induced characterization of orthology. For each parameter setting $i$, we define the full constraint graph $R^{i}$ where all gene pairs not predicted as orthologs are interpreted as paralogs. Typically, a graph $R^{-}$for a negative number $(-1$ or -2$)$ contains more orthology (and thus less paralogy) constraints than $R^{0}$, while the converse is true for a graph $R^{+}$. Combining two constraint graphs $R^{-}$and $R^{+}$consists in keeping only orthology and paralogy edges that are common to both, and completing the graph with unknown edges.

Table 1 summarizes the results on satisfiability and consistency with the Ensembl species tree $S$, obtained for each gene family and each parameter setting or combination. Among the 265 gene families, only 112 (42\%) produced at least one satisfiable full constraint graph and only $44(15 \%)$ produced such a graph which is also consistent with the Ensembl species tree. However, combining loose and strict parameter settings lead to much better results with at least $95 \%$ satisfiability and 56\% consistency with $S$. The partial orthology/paralogy constraints obtained from combinations correspond to about half of the constraints of a full graph, as illustrated by the last column of the table.

In order to get a rough idea of the accuracy of the obtained partial orthology/paralogy predictions for each gene family $\mathcal{G}$, we compared them with those resulting from the labeling of the Ensembl gene tree nodes as duplication and speciation nodes. An orthology disagreement refers to orthology predictions on the four combined graphs depicted in Table 1, that are rather inferred as paralogs from the Ensembl gene tree labeling.

Table 1 The results over 265 gene families from Ensembl.

\begin{tabular}{llll}
\hline & $\begin{array}{l}\text { \# satisfiable } \\
\text { families }\end{array}$ & $\begin{array}{l}\text { \# consistent } \\
\text { families }\end{array}$ & $\begin{array}{l}\text { \% constraints when } \\
\text { consistent }\end{array}$ \\
\hline-2 & $82(30.9 \%)$ & $30(11.3 \%)$ & \\
-1 & $44(16.6 \%)$ & $13(4.91 \%)$ & \\
0 & $26(9.81 \%)$ & $9(3.40 \%)$ & \\
+1 & $48(18.1 \%)$ & $14(5.28 \%)$ & \\
+2 & $55(20.8 \%)$ & $18(6.79 \%)$ & \\
$-2 /+2$ & $260(98.1 \%)$ & $172(64.9 \%)$ & $42.0 \%$ \\
$-2 /+1$ & $258(97.4 \%)$ & $172(64.9 \%)$ & $44.8 \%$ \\
$-1 /+1$ & $254(95.8 \%)$ & $149(56.2 \%)$ & $50.6 \%$ \\
$-1 /+2$ & $255(95.9 \%)$ & $157(59.2 \%)$ & $47.5 \%$ \\
\hline
\end{tabular}

The first five rows correspond to the full constraint sets obtained from PROTEINORTHO for the five classes of parameters. The last four rows correspond to the partial constraint sets obtained after combining two graphs over two types of parameters. The first column is the number of families for which the settings of PROTEINORTHO yielded a satisfiable graph, the second that number consistent with the Ensembl species tree. The last column shows the percentage of constraints that were not unknown when a consistent solution was found (which is $100 \%$ for the first five rows). 
A paralogy disagreement refers to the reverse situation. Overall, the orthology disagreement percentage is between $15.1 \%$ and $15.9 \%$ depending on the two classes of parameters combined. For paralogy disagreement, it varies between $11 \%$ and $17 \%$, depending on the 2 parameters combined $(-2 /+1$ and $-2 /+2$ were around $11.2 \%$ while $-1 /+1$ and $-1 /+2$ were around $17.4 \%$ ).

Notice that Ensembl annotates many duplication nodes as "dubious". If we ignore orthology disagreements caused by a dubious duplication node, the orthology disagreement percentage drops to an average of $5.0 \%$, strengthening the doubts on those duplication nodes.

\section{Conclusion}

In this work we have developed methods to assess the plausibility of a partial set of orthology and paralogy relationships between pairs of homologous genes. In particular, we showed how extending algorithms for the Graph sandwich problem can solve, in cubic time, the problems of satisfiability and consistency with a given species tree. In case of an unknown species tree, the complexity of the problem of verifying consistency with some species tree remains open. We have elaborated on the $P_{3}$ property of the constraint graph, which leads to an exponential branch-and- bound algorithm. It remains possible that this property could be used to create a more efficient method. While previous work consisted in verifying whether a full set of relationships was satisfiable or consistent, admitting uncertainty within these relationships makes it possible to bring the theory from [18] into practice, as current orthology (or paralogy) inference methods based on sequences cannot guarantee $100 \%$ accuracy in their predictions. We show how a confidence set of such predictions can be inferred using our methods and Proteinortho. A promising direction is to use such robust predictions to correct gene trees in case of disagreement.

\section{Availability}

Software is available at http://www-ens.iro.umontreal.ca/ lafonman/software.php.

\section{Competing interests}

The authors declare that they have no competing interests.

\section{Authors' contributions}

$\mathrm{ML}, \mathrm{NE}$ devised the proofs and algorithms and wrote the paper. ML implemented the software.

\section{Declarations}

Publication charges for this work was funded by the Natural Sciences and Engineering Research Council of Canada (NSERC) Discovery Grant and Fonds de Recherche Nature et Technologies of Quebec (FQRNT) B2 Grant This article has been published as part of BMC Genomics Volume 15 Supplement 6, 2014: Proceedings of the Twelfth Annual Research in
Computational Molecular Biology (RECOMB) Satellite Workshop on Comparative Genomics. The full contents of the supplement are available online at http://www.biomedcentral.com/bmcgenomics/supplements/15/S6.

\section{Published: 17 October 2014}

\section{References}

1. Ohno S: Evolution by gene duplication Berlin: Springer; 1970

2. Goodman M, Czelusniak J, Moore G, Romero-Herrera A, Matsuda G: Fitting the gene lineage into its species lineage, a parsimony strategy illustrated by cladograms constructed from globin sequences. Systematic Zoology 1979, 28:132-163.

3. Coghlan A, Coin JRL, Heriche J, Osmotherly L, Li R, T TL, Zhang Z, Bolund L, Wong G, Zheng W, Dehal P, J JW, Durbin R: TreeFam: a curated database of phylogenetic trees of animal gene families. Nucleic Acids Research 2006, 34:D572-580.

4. Schreiber F, Patricio M, Muffato M, Pignatelli M, Bateman A: TreeFam v9: a new website, more species and orthology-on-the-fly. Nucleic Acids Res 2013, 42:D922-D925.

5. Flicek P, Amode M, Barrell D, Beal K, Brent S, Carvalho-Silva D, Clapham P, Coates G, Fairley S, Fitzgerald S, Gil L, Gordon L, Hendrix M, Hourlier T, Johnson N, Kahari A, Keefe D, Keenan S, Kinsella R, Komorowska M, Koscielny G, Kulesha E, Larsson P, Longden I, McLaren W, Muffato M, Overduin B, Pignatelli M, Pritchard B, Riat H, Ritchie G, Ruffier M, Schuster M, Sobral D, Tang Y, Taylor K, Trevanion S, Vandrovcova J, White S, Wilson M, Wilder S, Aken B, Birney E, Cunningham F, Dunham I, Durbin R, FernA $A^{\sim}$ indezSuarez X, Harrow J, Herrero J, Hubbard T, Parker A, Proctor G, Spudich G, Vogel J, Yates A, Zadissa A, Searle S: Ensembl 2012. Nucleic Acids Res. 2012, 40:D84-D90.

6. Datta R, Meacham C, Samad B, Neyer C, Sjölander K: Berkeley PHOG: PhyloFacts orthology group prediction web server. Nucleic Acids Res. 2009, 37:W84-W89.

7. Pryszcz L, Huerta-Cepas J, Gabaldón T: MetaPhOrs: orthology nd paralogy predictions from multiple phylogenetic evidence using a consistencybased confidence score. Nucleic Acids Research 2011, 39:e32.

8. Tatusov R, Galperin M, Natale D, Koonin E: The COG database: a tool for genome-scale analysis of protein functions and evolution. Nucleic Acids Research 2000, 28:33-36.

9. Li L, Stoeckert CJ, Roos D: OrthoMCL: identification of ortholog groups for eukaryotic genomes. Genome Research 2003, 13:2178-2189.

10. Berglund A, Sjolund E, Ostlund G, Sonnhammer E: InParanoid 6: eukaryotic ortholog clusters with inparalogs. Nucleic Acids Research 2008, 36:D263-D266.

11. Lechner M, Findeib S, Steiner L, Marz1 M, Stadler P, Prohaska S: Proteinortho: detection of (co-)orthologs in large-scale analysis. BMC Bioinformatics 2011, 12:124

12. Waterhouse R, Zdobnov E, Tegenfeldt F, Li J, Kriventseva E: OrthoDB: the hierarchical catalog of eukaryotic orthologs in 2011. Nucleic Acids Research 2011, 39(Database):D283-D288.

13. Powell S, Forslund K, Szklarczyk D, Trachana K, Roth A, Huerta-Cepas J, Rattei T, Creevey C, Kuhn M, Jensen L, von Mering C, Bork P: eggNOG v4.0: nested orthology inference across 3686 organisms. Nucleic Acids Research 2013, 42:D231-D39.

14. Lafond M, Semeria M, Swenson K, Tannier E, El-Mabrouk N: Gene tree correction guided by orthology. BMC Bioinformatics 2013, 14(supp 15):S5

15. Lafond M, Swenson K, El-Mabrouk N: Models and algorithms for genome evolution Springer; 2013, Chap. Error detection and correction of gene trees.

16. Consortium TGO: Gene ontology: tool for the unification of biology. Nat Genet 2000, 25:25-29.

17. Hellmuth M, Hernandez-Rosales M, Huber K, Moulton V, Stadler P, Wieseke N: Orthology relations, symbolic ultrametrics, and cographs. J Math Biol 2013, 66(1-2):399-420.

18. Hernandez-Rosales M, Hellmuth M, Wieseke N, Huber K, Moulton V, Stadler P: From event-labeled gene trees to species trees. BMC Bioinformatics 2012, 13(Suppl. 19):56.

19. Hellmuth M, Wieseke N, Lechner M, Lenhof H, Middledorf M: Phylogenetics from paralogs 2014, [Unpublished manuscript].

20. Fitch WM: Homology. A personal view on some of the problems. TIG 2000, 16(5):227-231.

21. Golumbic M, Kaplan H, Shamir R: Graph Sandwich Problems. J Algorithms 1995, 19(3):449-473[http://dx.doi.org/10.1006/jagm.1995.1047] 
22. Semple C, Steel M: Phylogenetics. In Oxford Lecture Series in Mathematics and in Applications. Volume 24. [Oxford, UK: Oxford University Press]; 2003:119-120.

23. Aho A, Sagiv Y, Szymanski T, Ullman J: Inferring a tree from lowest common ancestors with an application to the optimization of relational expressions. SIAM J Comput 1981, 10:405-421.

doi:10.1186/1471-2164-15-S6-S12

Cite this article as: Lafond and El-Mabrouk: Orthology and paralogy constraints: satisfiability and consistency. BMC Genomics 2014

15(Suppl 6):S12.

Submit your next manuscript to BioMed Central and take full advantage of:

- Convenient online submission

- Thorough peer review

- No space constraints or color figure charges

- Immediate publication on acceptance

- Inclusion in PubMed, CAS, Scopus and Google Scholar

- Research which is freely available for redistribution

Submit your manuscript at www.biomedcentral.com/submit
C Biomed Central 